\title{
Constructing informative Bayesian map priors: a multi-objective optimisation approach applied to indoor occupancy grid mapping
}

\author{
Christina Georgiou* ${ }^{* \ddagger}$, Sean Anderson* and Tony Dodd*
}

January 27,2017

\begin{abstract}
The problem of Simultaneous Localisation And Mapping (SLAM) has been addressed in numerous ways with different approaches aiming to produce faster, more robust solutions that yield consistent maps. This focus, however, has resulted in a number of solutions that perform poorly in challenging real life scenarios. In order to achieve improved performance and map quality this paper proposes a novel method to construct informative Bayesian mapping priors through a multi-objective optimisation of prior map design variables defined using a source of prior information. This concept is explored for 2D occupancy grid SLAM, constructing such priors by extracting structural information from architectural drawings and identifying optimised prior values to assign to detected walls and empty space. Using the proposed method a contextual optimised prior can be constructed. This prior is found to yield better quantitative and qualitative performance than the commonly used non-informative prior, yielding an increase of over $20 \%$ in the $F_{2}$ metric. This is achieved without adding to the computational complexity of the SLAM algorithm, making it a good fit for time critical real life applications such as search and rescue missions.
\end{abstract}

SLAM, Bayesian estimation, informative priors

\section{Introduction}

Bayesian methods dominate the estimation algorithms used in Simultaneous Localisation and Mapping (SLAM) (Durrant-Whyte \& Bailey 2006), yet there has been very little research to date into how to effectively construct the Bayesian

\footnotetext{
*Department of Automatic Control and Systems Engineering, The University of Sheffield, Sheffield, S1 3JD, United Kingdom

${ }^{\dagger} \mathrm{PA}$ Consulting

${ }^{\ddagger}$ Corresponding author: PA Consulting Group, Cambridge Technology Centre, Melbourn, Herts, SG8 6DP, United Kingdom; e-mail: christina.georgiou@paconsulting.com
} 


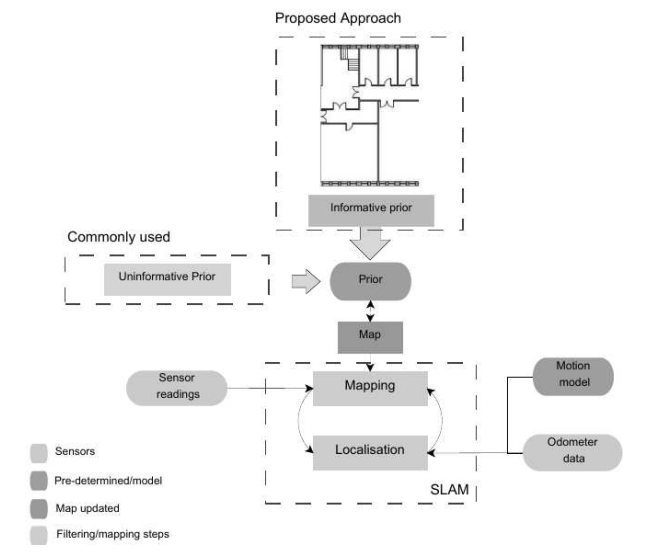

Figure 1: The structure of the SLAM problem: sensor data, prior information and motion model predictions are input to the SLAM algorithm that recursively maps the environment and localises the robot within it; architectural drawings and floor plans can be used to extract prior information. The commonly used Bayesian prior is constructed assuming there is no available information about the environment and so is uninformative. The proposed Bayesian prior is constructed using available information that is processed and placed in an optimised prior format.

map prior using available information. This is the case even though the use of priors is a key distinguishing feature of Bayesian estimation. The lack of attention given to the map prior can be contrasted to the enormous wealth of raw prior information that is readily available and untapped for most mapping and localisation scenarios, such as architectural drawings for buildings, city maps for the urban environment, geographical surveys for the wider outdoor environment and even pipe network maps for the underground environment. There exists an important and unsolved problem, therefore, in how to optimally synthesize raw prior information into a form that is suitable for robot navigation. This paper addresses this problem for 2D indoors occupancy grid SLAM by proposing a novel method to construct contextual priors that can improve the performance of robot navigation using architectural drawings and floor plans as a source of prior information (Figure 1).

Improving navigation performance by constructing and using meaningful priors can be very beneficial for time and safety critical applications, such as Urban Search And Rescue (USAR) missions. Using optimised priors to improve performance does not increase the computational complexity of the SLAM algorithm itself. There is only a small overhead cost of extracting appropriate structural information and constructing a prior map but that is a one-off operation that can be performed offline. Constructing an appropriate prior can also help produce better quality maps when a quick exploration is required by providing information about any unexplored areas. 
The idea of using prior information to improve the performance of robotics systems has been suggested for a number of systems that operate in real-world, challenging environments. Priors are used in self-driving cars to improve localisation (Maddern, Pascoe \& Newman 2015) and 3D semantic priors are used to interpret traffic lights (Barnes, Maddern \& Posner 2015). Prior knowledge can be incorporated in feature-based SLAM by using known landmarks (Burschka, Geiman \& Hager 2003). Implementations such as (Williams \& Reid 2010) use only a few known landmarks as points of reference to correct predictions and (Parsley \& Julier 2011) constrain the possible location of landmarks in GraphSLAM using prior information. As a result, the observed position error is reduced whilst maintaining the same or improved consistency compared to the no-prior solution (de la Puente \& Rodriguez-Losada 2014). Finally, skeletal SLAM (Milstein 2005) uses prior information about the shape of a building to construct more accurate occupancy grid maps.

In all of the above cases the use of prior information was found to be beneficial and yield improved performance in robot navigation. However, all of the methods reviewed above define the prior in an ad-hoc way, without using any particular method to choose prior probabilities from raw prior data. There is a gap, therefore, in constructing Bayesian map priors from available data using a formal, methodological approach, that can be optimised and repeated across different mapping scenarios. The main contribution of this paper is a novel method to construct contextual Bayesian map priors that improve map quality.

Georgiou2015 proposed the use of architectural drawings and floor plans as a source of prior information, presenting a novel method to extract wall and empty space locations from such drawings. The paper presented a method to extract information that can be used to construct the prior map, but did not explore how to construct and use such a prior map using detected walls and empty space. Building on (Georgiou, Anderson \& Dodd 2015), we aim to determine optimised values to assign to detected walls and empty space. The maps produced using these optimised values are tested in both simulation and experiment. The optimised Bayesian prior map is benchmarked here against the use of a non-informative prior, and the results demonstrate that the use of the optimised prior is hugely beneficial in the initial period of mapping and localisation, and retains an improvement in the final map. These results suggest that optimised Bayesian priors have great potential, especially in time-critical missions such as search and rescue.

The problem of developing a method for synthesizing optimal Bayesian map priors from raw data raises a number of key challenges, not least of which is how to measure map quality in an objective and quantitative way. This problem is addressed here by introducing, for the first time to this scenario, the approach of measuring map quality by the metrics of precision and recall. The definition and use of these metrics enables the optimisation of prior probabilities used to construct the map, so that they are not chosen in an ad-hoc way. However, precision and recall tend to conflict as optimisation objectives, which is a further challenge that is addressed here using multi-objective optimisation. The multiobjective optimisation does not produce a single optimal solution, but instead 
an optimal trade-off surface - a Pareto-optimal front. From the Pareto-front, the user can choose an appropriate trade-off between precision and recall to define the Bayesian prior map.

In summary, the following four steps are proposed as a solution to constructing informative Bayesian priors: 1. identify a source of prior information and a method to extract useful information from it; 2 . determine prior map design variables that affect final map quality; 3 . define quantitative measures of map quality - precision and recall; and 4. perform a multi-objective optimisation to identify prior probabilities that yield high performance metrics.

This paper is organised as follows. Section 2 gives an overview of the role of priors in SLAM and shows how using an optimised Bayesian prior can yield performance improvements. Section 3 presents the proposed strategy to construct informative priors and explains each aspect for the case study explored in this paper, indoors occupancy grid mapping. Section 3.1 presents the algorithm used to extract the location of walls from an architectural drawing or floor plan. Section 3.2 details a novel quantitative measure of map quality and Section 3.3 presents a multi-objective optimisation using a genetic algorithm to identify Pareto optimal design parameters. Section 4 presents the image test set used to demonstrate results in this paper and details the simulation used to obtain mapping results. Section 5 compares the maps produced in simulation using various shortlisted priors to determine which are optimal and presents the benefits of the proposed prior construction method. Finally, Section 6 validates the benefits of using an informative prior experimentally, both in a simulated robot course and in a large scale experiment.

\section{Bayesian priors in SLAM}

SLAM aims to have a robot explore and map an environment whilst simultaneously localising itself within it. The robot uses sensors to perceive the environment, a motion model or odometer to predict robot motion and it can also use a prior to incorporate information about the environment (Durrant-Whyte \& Bailey 2006). Each of the elements of the chosen implementation for this paper are presented in this section.

First the probabilistic formulation of SLAM is given, highlighting the role of the prior map. The implementation of choice, occupancy grid FastSLAM (Montemerlo, Thrun, Koller \& Wegbreit 2003), is then presented, showing the separation of the localisation and mapping tasks using Rao-Blackwellization, and the incorporation of prior information through the occupancy grid mapping algorithm is highlighted. This separation allows a study of mapping without the need to focus on performing localisation.

\subsection{Probabilistic formulation}

SLAM performs a Bayesian estimation to determine the robot's pose and a map of the environment given sensor readings, control inputs and the robot's initial 
pose. Formally, it aims to compute the joint posterior of the robot's pose $\mathbf{x}_{k}$ and the map m (Durrant-Whyte \& Bailey 2006)

$$
p\left(\mathbf{x}_{k}, \mathbf{m} \mid \mathbf{Z}_{0: k}, \mathbf{U}_{0: k}, \mathbf{x}_{0}\right) \propto \mathbf{A} \times p(\mathbf{m})
$$

for all times $k . \mathbf{x}_{k}$ is the state vector describing the robot's pose, $\mathbf{m}$ is the map of the environment (or a selection of landmarks for feature-based SLAM), $p(\mathbf{m})$ is the prior map, $\mathbf{Z}_{0: k}$ are the sensor observations, $\mathbf{U}_{0: k}$ the history of control inputs and $\mathbf{x}_{0}$ is the robot pose at time $k=0$. $\mathbf{A}$ is given by

$$
\mathbf{A}=\frac{p\left(\mathbf{z}_{k}, \mathbf{x}_{k} \mid \mathbf{m}\right)}{p\left(\mathbf{x}_{k}, \mathbf{m}\right)} \frac{p\left(\mathbf{x}_{k}, \mathbf{m} \mid \mathbf{Z}_{0: k-1}, \mathbf{U}_{0: k}, \mathbf{x}_{0}\right)}{p\left(\mathbf{z}_{k} \mid \mathbf{Z}_{0: k-1}, \mathbf{U}_{0: k}\right)}
$$

since the joint posterior in (1) can be written

$$
\begin{gathered}
p\left(\mathbf{x}_{k}, \mathbf{m} \mid \mathbf{Z}_{0: k}, \mathbf{U}_{0: k}, \mathbf{x}_{0}\right)= \\
p\left(\mathbf{z}_{k} \mid \mathbf{x}_{k}, \mathbf{m}\right) \frac{p\left(\mathbf{x}_{k}, \mathbf{m} \mid \mathbf{Z}_{0: k-1}, \mathbf{U}_{0: k}, \mathbf{x}_{0}\right)}{p\left(\mathbf{z}_{k} \mid \mathbf{Z}_{0: k-1}, \mathbf{U}_{0: k}\right)} \\
=\frac{p\left(\mathbf{z}_{k}, \mathbf{x}_{k} \mid \mathbf{m}\right)}{p\left(\mathbf{x}_{k}, \mathbf{m}\right)} p(\mathbf{m}) \frac{p\left(\mathbf{x}_{k}, \mathbf{m} \mid \mathbf{Z}_{0: k-1}, \mathbf{U}_{0: k}, \mathbf{x}_{0}\right)}{p\left(\mathbf{z}_{k} \mid \mathbf{Z}_{0: k-1}, \mathbf{U}_{0: k}\right)} \\
\propto \mathbf{A} \times p(\mathbf{m})
\end{gathered}
$$

indicating that the joint posterior is proportional to the prior probability $p(\mathbf{m})$. Therefore a choice of an optimised prior $p(\mathbf{m})$ can lead to a more accurate estimate of the joint posterior.

The aim is to estimate the robot pose for all times $k$ and the map of the environment given sensor readings, control inputs and a starting pose. SLAM is solved recursively, producing map and pose estimates at each time step.

In the probabilistic formulation of SLAM the tasks of localisation and mapping cannot be viewed separately. FastSLAM uses Rao-Blackwellization to separate localisation and mapping as discussed in the following section. Therefore FastSLAM allows the study of the effects of a prior map without the need to address the localisation aspect of SLAM.

\subsection{Occupancy grid FastSLAM formulation}

FastSLAM uses Rao-Blackwellization to decompose the SLAM problem into a robot localisation problem and a collection of landmark/map estimation problems that are conditioned on the robot trajectory estimate (Montemerlo et al. 2003), (Durrant-Whyte \& Bailey 2006).

$$
\begin{gathered}
p\left(\mathbf{X}_{0: k}, \mathbf{m} \mid \mathbf{Z}_{0: k}, \mathbf{U}_{0: k}, \mathbf{x}_{0}\right) \\
=p\left(\mathbf{m} \mid \mathbf{X}_{0: k}, \mathbf{Z}_{0: k}\right) p\left(\mathbf{X}_{0: k} \mid \mathbf{Z}_{0: k}, \mathbf{U}_{0: k}, \mathbf{x}_{0}\right)
\end{gathered}
$$

This separation of localisation and mapping allows the study of mapping priors without the need to explore localisation and is thus the implementation used in this paper. 
In this case the aim is to compute the joint posterior of the map and the complete robot trajectory $\mathbf{X}_{0: k}$ rather than the single pose $\mathbf{x}_{k}$. That is because landmarks conditioned on the trajectory are independent, allowing the factorisation shown in Equation 4. A particle filter can then be used to sample from the motion model and produce the proposal distribution but now the estimates for the landmark locations (conditioned on the robot trajectory estimate) are performed separately.

Since the pose and map estimates can be performed separately using RaoBlackwellization, the occupancy grid mapping algorithm can be used to calculate $p\left(\mathbf{m} \mid \mathbf{X}_{0: k}, \mathbf{Z}_{0: k}\right)$. The starting pose $\mathbf{x}_{0}$ is considered to be deterministic for the purposes of this paper, since for a known prior map we can measure pose $\mathbf{x}_{0}$ at $\mathrm{t}=0$ with respect to the prior map, similarly to the minimum covariance case in (Dissanayake, Newman, Clark, Durrant-Whyte \& Csorba 2001). Therefore prior information about the environment can only be incorporated into $p\left(\mathbf{m} \mid \mathbf{X}_{0: k}, \mathbf{Z}_{0: k}\right)$ through the Bayesian prior $p(\mathbf{m})$.

The occupancy grid mapping algorithm (Elfes 1989) is used in this paper to update the probabilities of occupancy of each grid cell in the environment. This representation is chosen since the prior map $p(\mathbf{m})$ is the map used at time $k=0$, which is then recursively updated as occupancy measurements are taken for map cells as the robot explores the environment.

The occupancy grid mapping algorithm (Elfes 1989) splits the environment to be mapped into a grid of cells and a prior probability is assigned to each cell. The log odd occupancy of each grid cell can be updated using

$$
\mathbf{l}_{k, i}=\text { InverseSensor Model }\left(\mathbf{m}_{i}, \mathbf{x}_{k}, \mathbf{z}_{k}\right)+\mathbf{l}_{k-1, i}-\mathbf{l}_{0, i}
$$

with

$$
\mathbf{l}_{k, i}=\log \frac{p\left(\mathbf{m}_{i} \mid \mathbf{Z}_{1: k}, \mathbf{x}_{1: k}\right)}{1-p\left(\mathbf{m}_{i} \mid \mathbf{Z}_{1: k}, \mathbf{x}_{1: k}\right)}
$$

A detailed derivation of Equation 5 is given in the Appendix.

The Bayesian priors for each cell, $p\left(\mathbf{m}_{i}\right)$, are incorporated through $\mathbf{l}_{0, i}$, the $\log$ odds prior for a given cell. When there is no available prior information a non-informative Bayesian prior is assigned to all grid cells, $p\left(\mathbf{m}_{i}\right)=0.5, i=$ $1, \ldots, N$. Most researchers use such a prior in order to produce solutions that do not depend on having prior knowledge of the environment (Durrant-Whyte \& Bailey 2006). Others claim that access to information such as detailed architectural drawings may be difficult (Kumar, Rus \& Singh 2004). Information such as floor plans for buildings like hospitals and offices is generally available, however, and can be used to extract useful information and construct SLAM priors.

Incorporating prior information does not add to the computational cost of running SLAM and only incurs a one-off cost of extracting prior information and constructing a prior. Since the posterior is proportional to the prior map (Equation 3) and given the recursive nature of SLAM, constructing an informative prior map $p(\mathbf{m})$ can help produce a more accurate map even if a quick exploration is performed. In the case of a quick exploration each grid cell may 
only be scanned once or twice, making the effect of the prior more significant. This is especially useful for time critical applications where the environment needs to be explored quickly, such as USAR missions.

\section{$3 \quad$ Proposed approach}

The proposed strategy to convert prior information to an informative Bayesian prior is presented in this section. This consists of the following steps

1. . A method to process a source of prior information such as an aerial image or architectural drawing to extract relevant prior information

2. . A set of prior map design variables that affect final map quality

3. . A set of metrics to assess the quality of the final map which can be used to select optimised design variables

4. . A multi-objective optimisation to identify design variables that yield high map quality metrics

The rest of this paper discusses each of those aspects for the case study of indoors occupancy grid mapping using architectural drawings and floor plans as a source of prior information.

\subsection{Extracting structural information from an architec- tural drawing}

In order to construct optimised priors an appropriate source of information needs to be identified. This can be an image such as an architectural drawing or floor plan for indoors SLAM or an aerial photograph or road network map for outdoors SLAM. These drawings or images then need to be processed to extract the location of occupied and unoccupied environment sections. For indoors environments, walls and empty space need to be identified. Similarly, if the source of prior information is a road map, roads and non-traversable areas can be detected. This paper focuses on the use of architectural drawings as a source of information and so this section presents a method to extract structural information from them to construct a prior map.

Structural information such as the location of building walls is required in order to construct an indoors prior map. Architectural drawings and floor plans, two-dimensional, top-down drawings of buildings containing structural information, suggested furniture and annotated text can be used to extract such information. These need to be processed to extract structural information in order to construct a meaningful prior map of the environment. Once the locations of structural elements such as walls have been determined, they can be used to assign appropriate prior values $p\left(\mathbf{m}_{i}\right)$ to all grid cells.

The algorithm used in this paper to extract prior information, Algorithm 1, was proposed in (Georgiou et al. 2015) and is designed to extract structural 


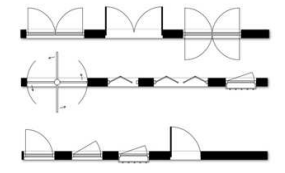

(a)

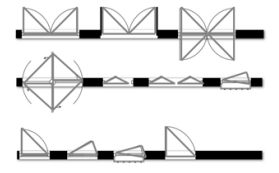

(b)

Figure 2: (a) Symbols for different types of doors (doors drawn using floorplanner.com) (b) The majority of door types can be approximated as isosceles triangles (drawn in grey).

information that can be used to construct robot priors. The main problem with processing drawings to extract this information is the lack of consistent representations between different drawings (Figure 2(a)). Depending on the architect and drawing method used, doors, walls and other elements can be represented in a different manner. Therefore, in order to yield reliable results for any drawing, the algorithm used to extract structural information needs to be independent of the drawing style.

The information the algorithm aims to extract is the location of walls which can be easily put in an occupancy grid format by assigning detected walls a low prior probability of being empty and detected space a high prior probability. Walls are represented as dark lines in the majority of drawings but have no other distinct geometric features. Given this variation between drawings, using methods such as line thickness to determine wall locations is unreliable. Instead of searching for walls directly, a feature with distinct geometric features and a clear relation to walls is detected. Doors are chosen for their distinctive shape and because they connect wall segments. Therefore if the location of doors in the image is found walls can also be detected.

There is no universally used symbol for all doors (Baden-Powell, Hetreed \& Ross 2011) and depending on the type of door (single, double, sliding, foldable, etc., Figure 2(a)) and the way the drawing was produced (different drawing software, hand-drawn) different representations can be used. However, all door symbols share a geometric characteristic: they can be approximated as isosceles triangles defined by three vertices and (usually) two edges/connected sides as shown in Figure 2(b). The proposed algorithm therefore searches for triangles in the image (as defined by their vertices) that fulfill the isosceles triangle criteria. This algorithm has a computational cost of $O\left(n^{3}\right)$ where $n$ is the number of Harris Corners detected in the image and it is dominated by the cost of finding the possible combinations $n \mathrm{Cr}=\frac{n !}{r(n-r) !}$ of $r=3$ out of $n$ detected Harris corners.

Once wall locations have been extracted from an architectural drawing, prior occupancy values $p\left(\mathbf{m}_{i}\right)$ need to be assigned to each grid cell $\mathbf{m}_{i}$ accordingly. Three parameters have to be chosen:

- Prior probability assigned to detected walls

- Prior probability assigned to detected empty space. 


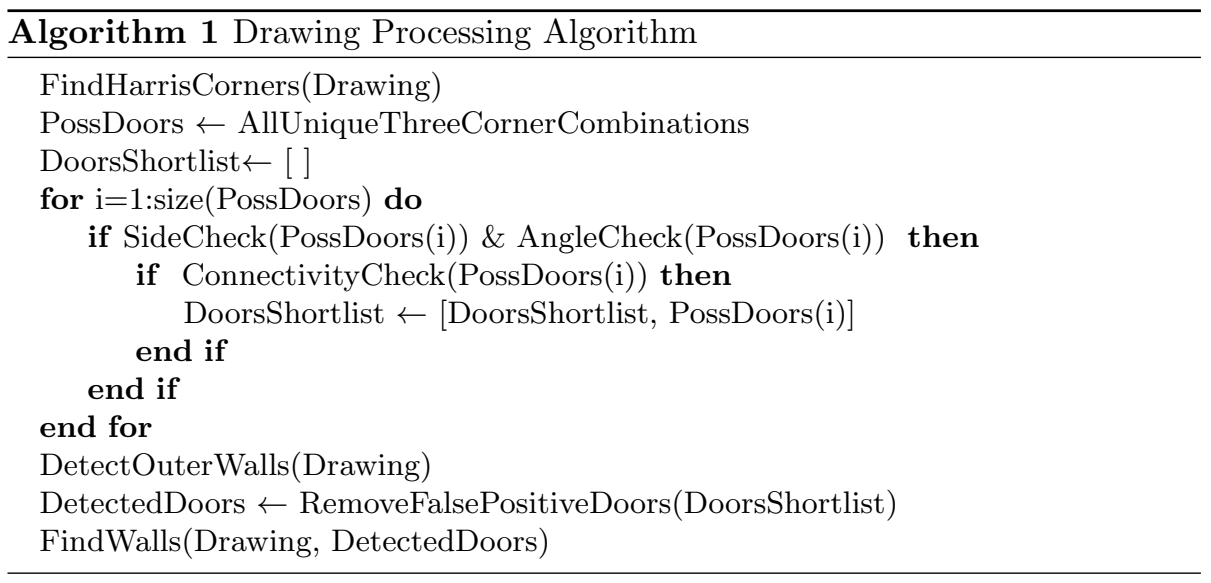

- Occupancy grid cell size (grid resolution)

Each grid cell $\mathbf{m}_{i}$ is assigned a Bayesian prior probability $p\left(\mathbf{m}_{i}\right)$ (Section 2.2). The following notation will be used throughout the rest of the paper. A high probability assigned to a grid cell will mean the cell has a high probability of being empty. Conversely, a low probability will signify a low probability that a cell is empty. If wall locations are known, cells that are located where walls were detected can be assigned lower prior probabilities, $p\left(\mathbf{m}_{i}^{\text {wall }}\right)<0.5$, and cells located where empty space was detected can be assigned higher prior probabilities, $p\left(\mathbf{m}_{i}^{\text {space }}\right)>0.5$. For brevity these two probabilities will be written $p_{\text {wall }}$ and $p_{\text {space }}$, where $p_{\text {wall }}$ is the prior probability assigned to grid cells that correspond to locations of detected walls in the architectural drawing and $p_{\text {space }}$ the prior probability assigned to cells corresponding to detected empty space in the drawing. This notation can be used since all grid cells $\mathbf{m}_{i}$ that correspond to occupied locations will be assigned a prior probability of $p_{\text {wall }}$ and those that correspond to empty space a prior probability $p_{\text {space }}$.

The alignment of grid cells and detected walls depends on the occupancy grid cell size. In all the results presented an appropriate grid cell size was chosen for all drawings used. The effects of using priors when coarser grids are used are discussed in the results section.

\subsection{Map quality assessment}

Using the method described in Section 3.1 walls and empty space can be detected in architectural drawings. Prior values of occupancy then need to be assigned to each grid square. In order to determine which $\left(p_{\text {wall }}, p_{\text {space }}\right)$ pair yields optimal performance the problem of assessing map quality is presented as a binary classification problem and a precision-recall analysis is performed.

The quality of a map produced by an occupancy grid mapping algorithm can be evaluated using a number of metrics proposed in the literature. Map consistency is a measure of performance but, according to (Mazuran, Tipaldi 
\& Stachniss 2014), there is no consistent notion of consistency and determining whether or not a SLAM map is consistent is still an open problem. Global consistency is often used to mean that the map produced agrees with the ground truth whereas local consistency refers to correctly aligning sensor scans locally. A measure for global consistency is proposed in (Mazuran et al. 2014) which uses the mismatch in the sensor data but this framework is tailored to SLAM using 2D laser sensors and can struggle in dynamic environments. (Collins, Collins \& Ryan 2007) compute a correlation between the produced map and ground truth but this tends to be computationally expensive.

In this paper we propose a metric that is easy to evaluate and that gives an indication of how successful the system was in detecting occupied and empty space in the environment. The use of the percentage of free and occupied cells identified correctly is proposed as a performance metric in (Grewe, Komar, Hohm, Lueke \& Winner 2012). The novelty of our proposed approach is the formulation of the assessment of the quality of an occupancy grid map as a classification problem where the aim is to correctly classify pixels as corresponding to occupied or unoccupied space. Therefore a precision-recall analysis can be used to evaluate the quality of the produced maps. Occupied locations in the environment can then be defined as true positives and empty space as true negatives. Any other objects detected can then be defined as false positives.

The chosen metrics are therefore detailed as follows (Davis \& Goadrich 2006):

$$
\begin{aligned}
& \text { - Precision }(\text { pre })=\frac{\text { TruePositives }}{\text { DetectedOccupiedSpace }} \\
& \text { - Recall }(\text { rec })=\frac{\text { TruePositives }}{\text { ActualOccupiedSpace }}
\end{aligned}
$$

with precision representing the percentage of correctly detected walls and recall representing the number of walls correctly detected out of all walls in the drawing.

Following these assessment criteria, each point in the robot map is tested against the true environment to determine whether it has been classified correctly. A simple way to perform this comparison in practice is to find the difference between the true environment image and the produced map to identify false positives and false negatives and thus calculate the above metrics. This is a simple but effective and computationally inexpensive method to assess quantitative performance.

Constructed priors are tested in a simulation which assumes perfect knowledge of the robot's pose so over or underestimating these values if the map and ground truth images are misaligned is not a problem. Therefore this method of comparing the map to ground truth to find the number of true/false positives/negatives is used to test the performance of maps produced using different priors.

If tests are conducted on a map pixel basis, coarse grids will assign occupied values to all cell pixels that correspond to detected occupied space, leading 
to a lower precision. A coarse grid would assign all cell pixels as occupied for a cell made up of mostly occupied space. For a large cell size this would result in incorrectly assigning many map pixels to occupied space, thus reducing precision. This would result in an area of misclassified points that are not strictly a classification error, just a limitation of an unsuitable grid resolution. In order to avoid penalising a system with an unsuitably coarse grid resolution, maps that perform well quantitatively are also tested qualitatively to ensure overall good performance.

While testing different prior maps for different drawings it was observed that the objectives of maximising precision and maximising recall are conflicting. Therefore selecting optimal prior values to maximise both objectives is not trivial and a multi-objective optimisation is proposed to determine optimised prior values.

\subsection{Multi-objective optimisation to optimise prior param- eters}

In order to test how the values for $\left(p_{\text {wall }}, p_{\text {space }}\right)$ that yield maximum precision and recall compare in a qualitative sense, maps can be constructed using the prior $\left(p_{\text {wall }}, p_{\text {space }}\right)$ values yielding maximum precision and recall. These can then be tested to assess the qualitative performance of maps produced using these prior values. If requirements for each metric are conflicting a multiobjective optimisation can be performed to determine pairs $\boldsymbol{\pi}=\left(p_{\text {wall }}, p_{\text {space }}\right)$ that yield both high precision and high recall. Having both precision and recall $>40 \%$ was empirically determined to be an acceptable threshold.

This multi-objective problem can be formulated as

$$
\begin{aligned}
\min _{\boldsymbol{\pi}} F(\boldsymbol{\pi}) & =\left[\begin{array}{l}
\left.f_{\text {pre }}(\boldsymbol{\pi}) \quad f_{\text {rec }}(\boldsymbol{\pi})\right] \\
\text { subject to } C
\end{array}=\left\{\begin{array}{l}
0.1 \leq \boldsymbol{\pi}<1 \\
f_{\text {pre }}(\boldsymbol{\pi})<2.5 \\
f_{\text {rec }}(\boldsymbol{\pi})<2.5
\end{array}\right.\right.
\end{aligned}
$$

where $\boldsymbol{\pi}=\left(p_{\text {wall }}, p_{\text {space }}\right), f_{\text {pre }}(\boldsymbol{\pi})=\frac{1}{\text { pre }}$ and $f_{\text {rec }}(\boldsymbol{\pi})=\frac{1}{\text { rec }}$ (so $f_{\text {pre }}(\boldsymbol{\pi})$, $f_{\text {rec }}(\boldsymbol{\pi})<2.5 \Leftrightarrow$ pre, rec $\left.>40 \%\right)$.

In order to perform this multi-objective optimisation a Pareto-based method was chosen since the relative importance of the objectives is unclear (Giagkiozis, Purshouse \& Fleming 2015) and a controlled elitist genetic algorithm (Deb 2001) (a variant of NSGA-II (Deb, Pratap, Agarwal \& Meyarivan 2002)) was used.

In this minimisation problem a decision vector $\hat{\boldsymbol{\pi}}$ with $\hat{\boldsymbol{\pi}} \in C$ is Pareto optimal if there is no other $\boldsymbol{\pi} \in C$ for which $f_{i}(\boldsymbol{\pi}) \leq f_{i}(\hat{\boldsymbol{\pi}}), \forall i$ and at least one $f_{i}(\boldsymbol{\pi})<f_{i}(\hat{\boldsymbol{\pi}})$ for $i=1, \ldots, K$ where $K$ is the number of functions in $F(\boldsymbol{\pi})$. In this case the decision vector $\hat{\boldsymbol{\pi}}$ is said to Pareto-dominate vector $\boldsymbol{\pi}$. If only the second condition is met the solution is considered weakly Pareto optimal. The 
multi-objective optimisation solver used aims to find a subset of Pareto optimal solutions which is referred to as the Pareto front (Giagkiozis et al. 2015).

The concept of Pareto dominance is applied in order to use a genetic algorithm to solve this multi-objective problem. First the objective function is evaluated for each individual in the population $\Pi$. Non-dominated individuals, $\boldsymbol{\Pi}_{\text {nond }}$ are then found and removed from the population. This process is repeated until all non-dominated individuals $\boldsymbol{\Pi}_{n o n d}$ have been identified.

In order to obtain recall and precision variables and perform the multiobjective optimisation a simulation environment was devised as detailed in the next section.

\section{Simulation setup}

A simulation was used to produce maps using different $\left(p_{\text {wall }}, p_{\text {space }}\right)$ pairs. A simulation allows the testing of a large number of possible prior values to assign to $\left(p_{\text {wall }}, p_{\text {space }}\right)$. Thus the multi-objective optimisation can be performed without the need to obtain mapping results for different buildings using a robot which would be very time consuming. The drawing test set, simulation used and assumptions made are discussed in this section.

\subsection{Drawing test set selection}

A number of different drawings were tested using the simulation environment described in this section. A version of each drawing containing only structural information was created by hand and used as the ground truth. Two floor plans, drawings (a) and (b) in Figure 4A, are presented, with drawing (a) containing labeling and drawing (b) containing suggested furniture. Drawings (c), (d) and (e) in Figure 4A, are sections of an architectural drawing of a University of Sheffield Engineering building. These sections were chosen because they are a representative sample of different outlines and commonly observed elements. Drawing (d) contains only doors and wall segments making it an easy drawing to process and drawings (c) and (e) are quite challenging to process, containing stairs, text, walls of varying width and, in the case of drawing (e), a door at an angle. These were chosen as a representative sample of common elements and configurations found in architectural drawings and floor plans.

\subsection{Occupancy grid mapping simulation}

In order to test the effects of using architectural priors on map quality a simulation environment was created in Matlab. In this simulation the robot pose is assumed to be known and accurate. Given the separation of localisation and mapping using Rao-Blackwellization in FastSLAM (Section 2.2), mapping can be studied separately by providing deterministic pose values instead of using

Monte Carlo localisation to produce pose estimates. Mathematically, instead of using Equation 4 and producing estimates of both the map and trajectory 
for each particle we assume $p\left(\mathbf{X}_{0: k} \mid \mathbf{Z}_{0: k}, \mathbf{U}_{0: k}, \mathbf{x}_{0}\right)$ is known and hence only one map needs to be updated by determining $p\left(\mathbf{m} \mid \mathbf{X}_{0: k}, \mathbf{Z}_{0: k}\right)$ using the occupancy grid mapping algorithm (Elfes 1989).

The robot is modeled as a point moving through space, with the robot state $\mathbf{x}_{k}$ at each time step $k$ given by

$$
\mathbf{x}_{k}=(x, y, \theta)
$$

where $(x, y)$ give the robot position in Cartesian coordinates and $\theta$ the robot orientation. The distance sensor on the robot is modeled as an ultrasound sensor with a conical field of view defined by an angle $\phi$ and range $r$.

The occupancy grid mapping algorithm (Elfes 1989) is used to update the $\log$ odds of occupancy $\mathbf{l}_{k, i}$ for each grid cell at each time step (Section 2.2). The probabilities of occupancy can be retrieved from $\mathbf{l}_{k, i}$ using

$$
p\left(\mathbf{m}_{i} \mid \mathbf{Z}_{1: k}, \mathbf{x}_{1: k}\right)=1-\frac{1}{1+\exp \left(\mathbf{l}_{k, i}\right)}
$$

The inverse model used is a multiplier, multiplying true occupancy values by a factor of 0.9 to introduce some uncertainty. The code used to update the occupancy grid map was also used to process real sensor and robot pose data collected during experiments.

When prior information is available $p\left(\mathbf{m}_{i}\right)$ can be assigned a different prior value based on whether a wall or empty space was detected for grid cell $i$ during the drawing processing stage as discussed in Section 3.1. The results obtained using this simulator and performing the multi-objective optimisation to determine optimal prior values $\left(p_{\text {wall }}, p_{\text {space }}\right)$ are presented in the next section.

\section{Simulation Results}

This section presents the results obtained using the simulator discussed in Section 4.2 , testing different prior values to assign to detected walls $p_{\text {wall }}$ and to detected empty space $p_{\text {space }}$. The results of the multi-objective optimisation performed to determine the $\left(p_{\text {wall }}, p_{\text {space }}\right)$ pair that yields optimised precision and recall are also presented. Finally the map produced using the proposed optimised prior is compared to the map produced using a non-informative prior and is found to yield an increase in the $F_{2}$ metric by at least $20 \%$.

\subsection{Designing contextual priors that optimise conflicting performance metrics}

In order to evaluate the effect of different priors, possible combinations of prior values $\left(p_{\text {wall }}, p_{\text {space }}\right)$ between 0.1 and 1 , multiplied by a factor of 0.9 to avoid values too close to 1 , were examined and linear interpolation was used to produce continuous values. Figure $3 \mathrm{C}$ shows colour maps of the precision and recall metrics for possible combinations of $p_{\text {wall }}$ and $p_{\text {space }}$ for the drawing in 
Figure 3A. Figure 3B shows the final maps produced using the prior values that yield maximum precision and those that yield maximum recall.

A higher precision ensures that it is unlikely free space will be incorrectly identified as occupied and a higher recall ensures that the building structure is detected. Figure 3C shows that the aims of having high precision and also maintaining a high recall are conflicting since pairs that yield high precision tend to yield low recall and vice versa. This problem of determining a suitable pair of $\left(p_{\text {wall }}, p_{\text {space }}\right)$ that ensures high precision and high recall $(>40 \%$ was empirically determined to be an appropriate threshold) is therefore a multiobjective optimisation problem.

In order to perform this multi-objective optimisation a controlled elitist genetic algorithm (Deb 2001) (a variant of NSGA-II (Deb et al. 2002)) was used. The Pareto front for the drawing in Figure 3A can be seen in Figure 3C and the final map produced using one of the Pareto optimal prior values is shown in Figure 3E. This drawing was chosen because, due to the level of detail including labeling, walls at an angle and multiple rooms, it highlights the qualitative difference in performance between Figure 3B and Figure 3E.

The map shown in Figure 3E avoids detecting thicker walls or missing doors as can happen using the prior values that optimise recall, Figure $3 \mathrm{~B}$, and also avoids missing most of the walls as is done using the prior values that yield maximum precision, Figure 3B.

\subsection{Multi-objective optimisation to determine globally op- timal contextual priors}

Figure $4 \mathrm{~B}$ shows the precision and recall colour maps for all representative drawings in Figure 4A. The shape of the colour maps for precision and recall does not vary greatly between drawings, other than the fact that the region of values that yield good performance is larger/smaller for different drawings. These results indicate that a globally optimal region of $\left(p_{\text {wall }}, p_{\text {space }}\right)$ values that result in high precision can be identified.

The locations of maximum precision for three different grid resolutions are shown in Table 1. Maximum values are consistently observed for $p_{\text {wall }}$ values between 0.1 and 0.3 and $p_{\text {space }}=0.3$ regardless of the drawing and grid resolution with the exception of drawing (a) for a 10x10 grid cell.

The values that yield optimal recall can be seen in Table 2 . These values are in most cases what one would intuitively expect to be optimal: a high value for $p_{\text {space }}$ and a lower value for $p_{\text {wall }}$. For the architectural drawing sections, optimised values were found to be $p_{\text {space }}=1$ with $p_{\text {wall }}$ between 0.1 and 1 . Both recall and precision are important to ensure a high quality map.

As shown in Tables 1 and 2 the values that yield maximum precision do not correspond to values that yield maximum recall and vice versa. Moreover, as shown in Figure 4B regions of high precision correspond to regions of low recall and vice versa. Therefore multi-objective optimisation is used to identify prior values that optimise both precision and recall. 
A Original architectural drawing

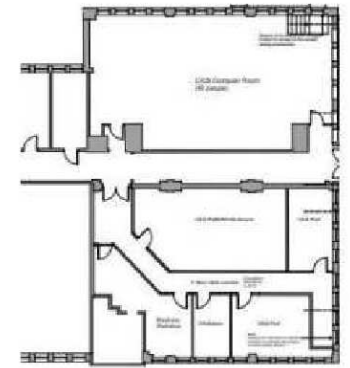

C Precision and recall metrics

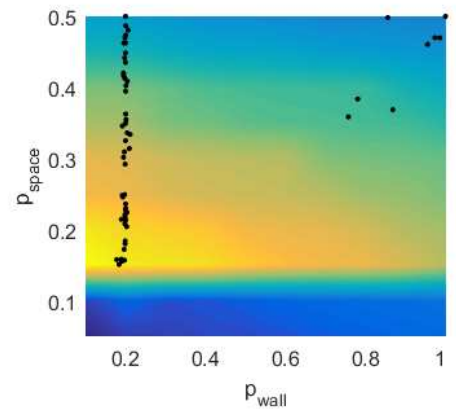

D Multi-objective optimisation: Pareto front

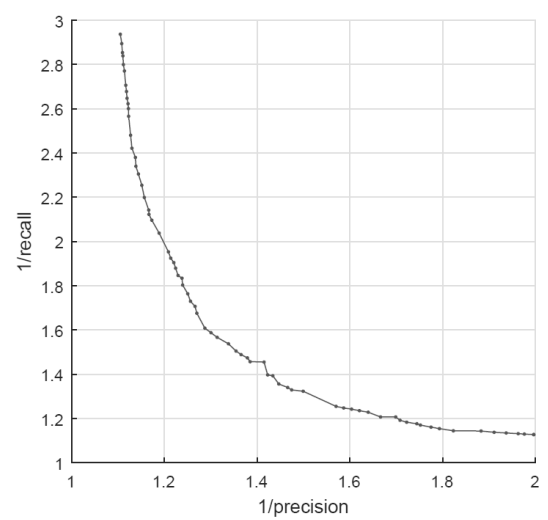

B Maps produced using a prior that maximises a single objective
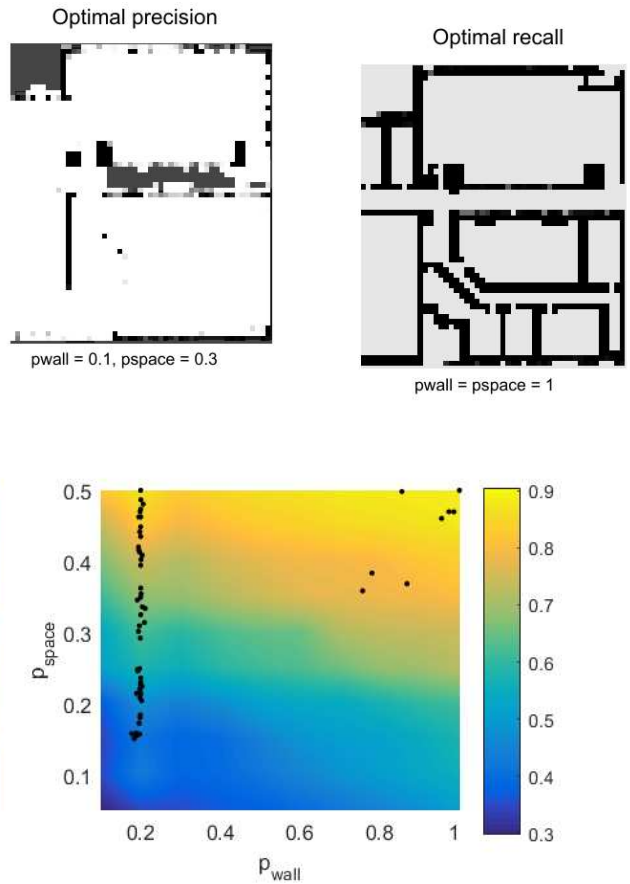

E Map produced using prior optimising conflicting objectives

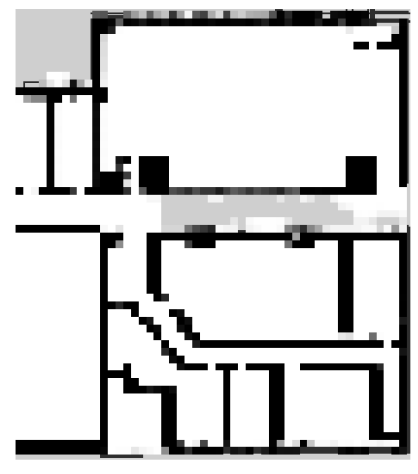

pwall $=0.2$, pspace $=0.9$

Figure 3: Multi-objective optimisation overview: A is the architectural drawing used to extract prior information; B shows the maps produced using prior values that yield maximum precision and maximum recall, neither of which is qualitatively optimal; $\mathrm{C}$ shows the pre and rec colour maps for all combinations of $\left(p_{\text {wall }}, p_{\text {space }}\right)$ between 0.1 and 1 , with Pareto optimal values shown as black points; D shows the Pareto front produced by the multi-objective otpimisation and $\mathrm{E}$ shows the map produced using the Pareto optimal proposed prior values $p_{\text {wall }}=0.2, p_{\text {space }}=0.9$. 
(a)

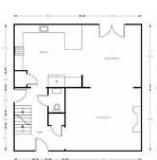

(b)

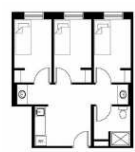

(c)

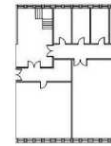

(d)

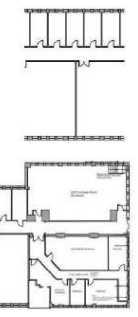

A

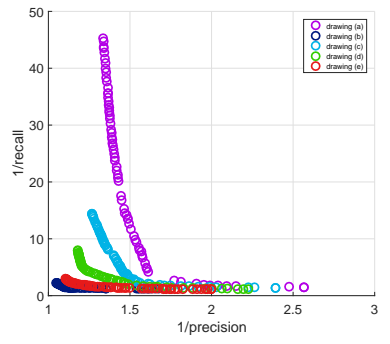

C
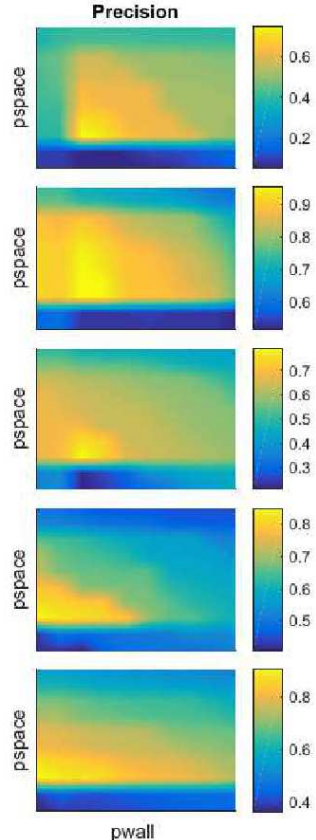

pwall

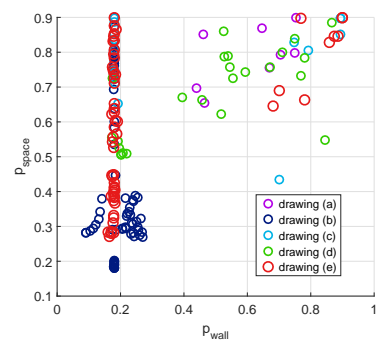

D
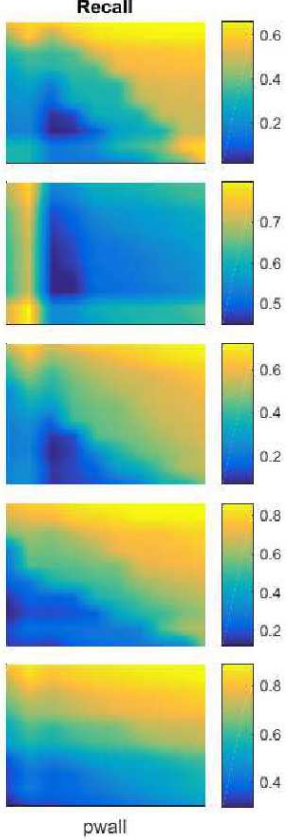

B

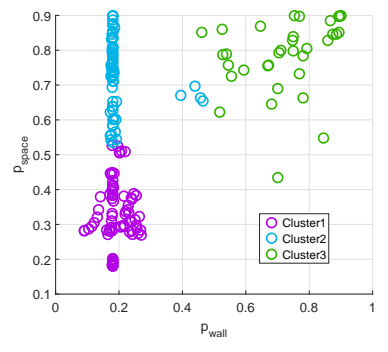

E

Figure 4: Multi-objective optimisation results for all drawings: A Drawings used to extract prior information; B Interpolated precision and recall colour maps for the five drawings; C Pareto fronts for all drawings, with a number of common solutions across drawings meaning there is a set of Pareto optimal $\left(p_{\text {wall }}, p_{\text {space }}\right)$ pairs that yield good performance for all drawings; D Pareto optimal $\left(p_{\text {space }}, p_{\text {walls }}\right)$ pairs plotted for all drawings; E Clusters detected within Pareto optimal solutions using the k-means clustering algorithm. 


\begin{tabular}{|c|c|c|}
\hline \multicolumn{3}{|c|}{ Grid cell $3 \times 3$} \\
\hline Drawing & Max pre \% & $\left(p_{\text {wall }}, p_{\text {space }}\right)$ \\
\hline $\mathrm{a}$ & 98.49 & $(0.3,0.3)$ \\
\hline b & 99.42 & $(0.3,0.3)$ \\
\hline $\mathrm{c}$ & 91.77 & $(0.3,0.3)$ \\
\hline d & 95.56 & $(0.1,0.3)$ \\
\hline $\mathrm{e}$ & 93.77 & $(0.3,0.3)$ \\
\hline \multicolumn{3}{|c|}{ Grid cell 5x5 } \\
\hline $\mathrm{a}$ & 75.00 & $(0.3,0.3)$ \\
\hline $\mathrm{b}$ & 95.36 & $(0.3,0.3)$ \\
\hline $\mathrm{c}$ & 79.00 & $(0.3,0.3)$ \\
\hline d & 84.94 & $(0.1,0.3)$ \\
\hline $\mathrm{e}$ & 90.51 & $(0.1,0.3)$ \\
\hline \multicolumn{3}{|c|}{ Grid cell 10x10 } \\
\hline $\mathrm{a}$ & 48.00 & $(0.5,0.6)$ \\
\hline $\mathrm{b}$ & 89.14 & $(0.1,0.3)$ \\
\hline $\mathrm{c}$ & 77.42 & $(0.1: 0.2,0.3: 05)$ \\
\hline $\mathrm{d}$ & 92.06 & $(0.1,0.3)$ \\
\hline $\mathrm{e}$ & 92.68 & $(0.1,0.3)$ \\
\hline
\end{tabular}

Table 1: Maximum precision calculated for each of the drawings in Figure 4, grid cell size given in number of map pixels.

Multi-objective optimisation using the controlled elitist genetic algorithm (Deb 2001) (a variant of NSGA-II (Deb et al. 2002)) discussed in Section 5.1 was performed for all five drawings. Figure $4 \mathrm{~B}$ shows the precision and recall interpolated colour maps for the five drawings used to generate the Pareto fronts in Figure 4C. Figure 4C shows the Pareto optimal fronts for all drawings and floor plans. There is a region where the Pareto optimal fronts for all drawings overlap, meaning there are Pareto optimal sets of $\left(p_{\text {wall }}, p_{\text {space }}\right)$ values that yield good performance regardless of the type of drawing used. Only solutions that fall within set $C$ as defined in Section 3.3 are of interest.

In the precision plots of Figure $4 \mathrm{~B}$ we observe higher values for $p_{\text {wall }}=0.2$. That is because 0.2 is the lowest probability value for which the log odds value is in the linear section of the log odds plot, Figure 5 . Therefore it is the lowest value we can assign to detected walls that avoids the region close to the asymptote near 0 .

In Tables 1 and 2 lower prior values for both $p_{\text {wall }}$ and $p_{\text {space }}$ do not yield optimal recall. This seems counter-intuitive given that assigning all cells a low prior probability would be expected to yield a low precision but high recall. However, low $p_{\text {wall }}$ and $p_{\text {space }}$ can result in assigning high probabilities of being empty to grid cells that contain mostly occupied space.

Using equation 5 , for low $p_{\text {wall }}$ and $p_{\text {space }}$, if a cell contains both occupied and empty space the simulator assigns an inverse sensor model value of 


\begin{tabular}{ccc}
\hline \multicolumn{3}{l}{ Grid cell $3 \times 3$} \\
\hline Drawing & Max rec $\%$ & $\left(p_{\text {wall }}, p_{\text {space }}\right)$ \\
\hline a & 80.08 & $(1,0.2)$ \\
b & 82.77 & $(0.2,0.1: 0.2)$ \\
c & 69.43 & $(0.1,1)$ \\
d & 82.70 & $(0.7: 1,1)$ \\
e & 83.05 & $(0.5: 1,1)$ \\
\hline \multicolumn{4}{l}{ Grid cell $5 \times 5$} & \\
\hline a & 66.09 & $(1,1)$ \\
b & 79.71 & $(0.2,0.2)$ \\
c & 72.05 & $(1,1)$ \\
d & 86.00 & $(0.4: 1,1)$ \\
e & 88.68 & $(0.9: 1,1)$ \\
\hline Grid cell & $10 \times 10$ & \\
\hline a & 66.53 & $(1,1)$ \\
b & 73.42 & $(0.2,1)$ \\
c & 86.91 & $(1,1)$ \\
d & 91.69 & $(0.4: 0.9,1)$ \\
e & 95.41 & $(0.2,1)$ \\
\hline
\end{tabular}

Table 2: Maximum recall calculated for each of the drawings in Figure 4, grid cell size given in number of map pixels.

$$
\begin{array}{r}
\text { InverseSensorModel }\left(\mathbf{m}_{i}, \mathbf{x}_{k}, \mathbf{z}_{k}\right)= \\
\log \operatorname{Odds}\left(0.9 \times \frac{p e l_{\text {empty }}}{N_{\text {cell }}}\right)
\end{array}
$$

where pelempty are the number of pixels corresponding to detected empty space in a given cell and $N_{\text {cell }}$ the number of map pixels in a grid cell. A low prior $p_{\text {space }}<0.2$ would result in $l_{0, i}<-1.4$. For a cell containing fewer than $70 \%$ pixels, corresponding to detected occupied space InverseSensor Model $\left(\mathbf{m}_{i}, \mathbf{x}_{k}, \mathbf{z}_{k}\right)>$ 0.4 . Therefore, using equation $5, l_{k, i}>2 \times(k-1) \times 2.8$, which corresponds to a probability greater than 0.94 , incorrectly mapping this as an empty cell.

The Pareto optimal solutions for all drawings were plotted as shown in Figure 4D. The k-means clustering method (Hartigan \& Wong 1979), (Arthur \& Vassilvitskii 2007) was then used to assign each point to clusters and the results are shown in Figure 4E.

In order to identify an optimal cluster of solutions, representatives from each cluster were tested in terms of qualitative performance. Solutions in Cluster 2, the light blue cluster in Figure $4 \mathrm{E}$, have high values of both $p_{\text {wall }}$ and $p_{\text {space }}$. That is the region where maximum recall solutions lie, as shown in Table 2. However, solutions within this region do not provide optimal qualitative results as shown in Figure 3B. Solutions that fall in Cluster 1, the purple cluster in 


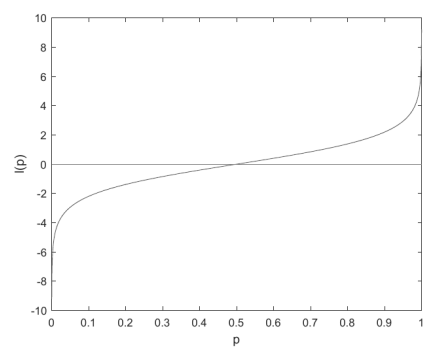

Figure 5: The log odds plot for probability values $0-1$. In the region $0.2-0.8$ the log odds show linear behaviour, whereas for values outside that range the resulting log odds value increases rapidly

Figure $4 \mathrm{E}$ have low values of both $p_{\text {wall }}$ and $p_{\text {space }}$ and thus correspond to the maximum precision solutions as shown in Table 1. These were also shown to produce non-optimal qualitative results as discussed in Section 5.1 and shown in Figure 3B. Therefore the optimal solution lies in Cluster 3, the green cluster.

The values in the green cluster are those that intuitively would be expected to perform well: a low $p_{\text {wall }}$ and a high $p_{\text {space }}$. Converting these back to discrete values, the possible solutions to investigate are the pairs

$$
\text { Shortlist }=\left\{\begin{array}{l}
p_{\text {wall }}=0.2,0.6 \leq p_{\text {space }} \leq 1 \\
0.4 \leq p_{\text {wall }} \leq 0.5,0.7 \leq p_{\text {space }} \leq 0.8
\end{array}\right.
$$

The maps produced using each of these pairs were compared in terms of visual quality and the best $\left(p_{\text {wall }}, p_{\text {space }}\right)$ combination out of the green cluster points in terms of qualitative performance was found to be $p_{\text {wall }}=0.2, p_{\text {space }}=$ 0.9 , Figure 6 . The next sections discuss the benefits of using an informative prior and compare the maps produced using the proposed informative prior to those produced using an uninformative prior to quantify the improvement in performance.

\subsection{Effects of sensor model on optimised prior values}

The proposed optimised prior values were obtained for an optimisation using the simple sensor model described in Section 4.2. The effect of selecting different sensor models is explored in this section. Different sensor models assign a different probability of occupancy to detected free and occupied space, setting $p_{\text {free }}$ to detected empty space cells and $p_{\text {occupied }}$ to cells corresponding to occupied space. More complex models might use a normal distribution centred around the location of a detected obstacle/detected empty space cell for $p_{\text {occupied }}$ and $p_{\text {free }}$ (Pirker, Rüther, Bischof \& Schweighofer 2011).

The log odds representation is used for the map update, with the initialisa- 


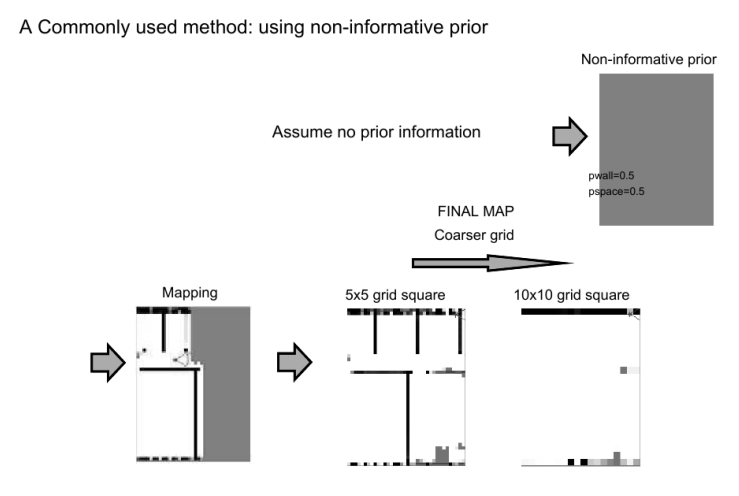

B Proposed method: using proposed informative prior
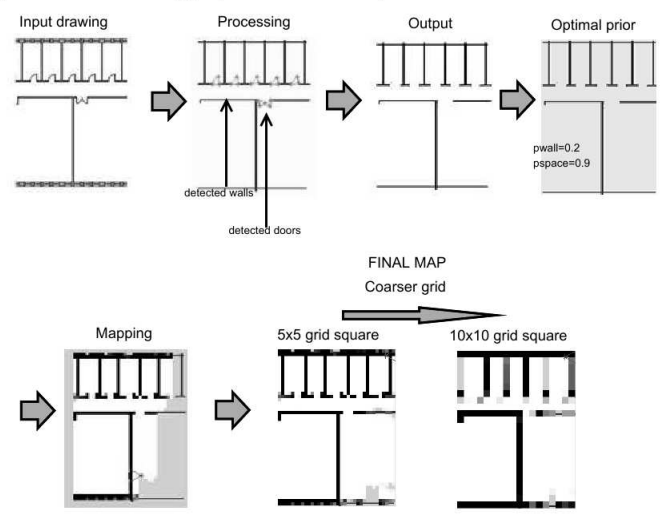

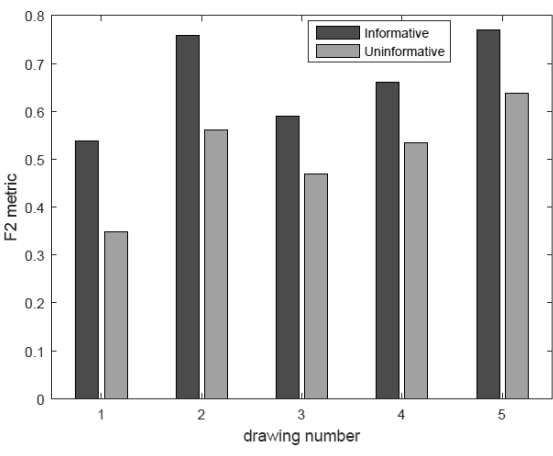

$\mathrm{C}$

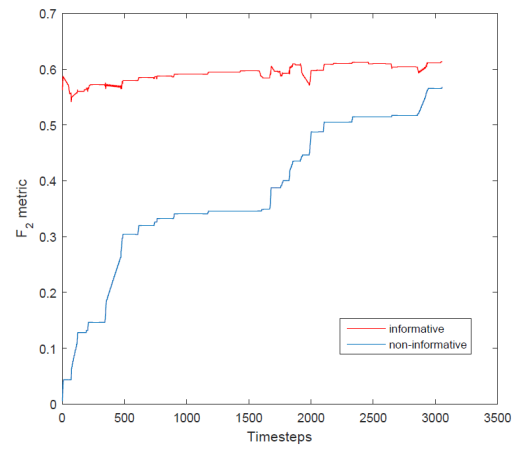

$\mathrm{D}$

Figure 6: Comparison of map produced using the proposed optimised prior and the map produced using a non-informative prior: A Maps produced using a noninformative prior for two different grid resolutions; B Processing and converting a drawing to an optimised prior and using it to produce maps for two different grid resolutions; C A quantitative comparison of the maps produced using an informative and a non-informative prior using the $F_{2}$ metric; D Evolution of $F_{2}$ metric with time when using an informative and a non-informative prior for the environment shown in A and B. 
tion of $\mathbf{l}_{k, i}=\mathbf{l}_{0, i}$. Using equation 5 , this leads to the update rule

$$
\begin{gathered}
\mathbf{l}_{1, i}=\text { InverseSensorModel }\left(\mathbf{m}_{i}, \mathbf{x}_{1}, \mathbf{z}_{1}\right) \\
\mathbf{l}_{2, i}=2 \times \text { InverseSensorModel }\left(\mathbf{m}_{i}, \mathbf{x}_{2}, \mathbf{z}_{2}\right)-\mathbf{l}_{0, i} \\
\vdots \\
\mathbf{l}_{k, i}=k \times \text { InverseSensorModel }\left(\mathbf{m}_{i}, \mathbf{x}_{k}, \mathbf{z}_{k}\right)-(k-1) \times \mathbf{l}_{0, i}
\end{gathered}
$$

for the $i$ th cell at the $k$ th iteration. Therefore, to obtain a larger final map probability for empty cells we need $\mathbf{l}_{k, i}>0$ corresponding to a probability of occupancy $\mathbf{p}_{m_{i}}^{k}>0.5$, Figure $5 . \mathbf{l}_{k, i}>0$ would require

$$
\text { InverseSensor Model }\left(\mathbf{m}_{i}, \mathbf{x}_{k}, \mathbf{z}_{k}\right)>\mathbf{l}_{0, i}
$$

for a large $k$. Therefore the probability assigned by the sensor model to detected free space should be greater than the prior value assigned to empty space $p_{\text {free }}>p_{\text {space }}$. Conversely, the value assigned to detected occupied space by the sensor model should be smaller than the prior, $p_{\text {occupied }}<p_{\text {wall }}$.

\subsection{Benefits of using an optimised informative prior}

Using an optimised informative prior allows for improved performance when a robot quickly explores an area while operating in a time-critical mission such as USAR, for example. In the mapping example presented in Figure 6 the robot has not fully explored the environment. In such cases using a prior has the added benefit of providing information about building sections even if areas are unexplored. Moreover, for coarse grid resolutions the commonly used uninformative prior produces maps of very low quality whereas the proposed optimised prior of $p_{\text {wall }}=0.2, p_{\text {space }}=0.9$ produces maps that give an overview of building structure, as shown in Figure 6. Therefore if a quick and computationally cheaper exploration is required the proposed optimised prior yields significantly better maps in terms of visual quality, identifying the majority of building walls.

The maps produced using the proposed prior perform better in a quantitative sense as shown in Figure 6C. Given the multi-objective nature of the optimisation, merely comparing the values of precision and recall for maps produced using the informative and non-informative prior does not provide enough information. In order to overcome this problem the $F_{2}$ metric (Powers 2011) is used to allow a comparison of the precision and recall combination rather than individual values for each drawing.

$$
F_{2}=5 \times \frac{\text { pre } \times r e}{(4 \times p r e)+r e c}
$$

Recall is favoured over precision since it represents the percentage of correctly detected walls and thus affects the map outline more. As shown in Figure $6 \mathrm{C}$ using an informative prior yields an increase in $F_{2}$ measure of at least $20 \%$ over the uninformative prior. Moreover, it is worth noting that using an informative prior maintains a high $F_{2}$ throughout and from the early stages 
of exploration (Figure 6D) greatly outperforming the map produced using the non-informative prior.

The overall method proposed in this paper to process an architectural drawing or floor plan, extract structural information and use it to construct an optimised prior and thus an improved map is shown in Figure 6B.

\section{$6 \quad$ Experimental results}

In order to verify the results obtained using simulated data, distance data were collected using a turtlebot mounted with a Microsoft Kinect sensor running ROS. Two sets of experiments were conducted

- A small scale experiment in a constructed course, aiming to simulate a building, Figure 7A. The robot pose was measured perfectly by hand.

- A large scale experiment in a floor of a University of Sheffield Engineering building. In this case we used Adaptive Monte Carlo Localisation and the extracted prior to produce estimates of the robot pose.

In both cases the occupancy grid Matlab code presented in Section 4.2 was used to update the map.

\subsection{Constructed robot course}

A known, accurate robot pose was used at each time step and the occupancy grid map update in the Matlab simulator proposed in Section 4.2 was used to process the data collected using the Kinect sensor.

An informative prior map produced using perfectly extracted walls and assigning the proposed prior values $p_{\text {wall }}=0.2, p_{\text {space }}=0.9$ was used, Figure 7B. The maps produced using the proposed informative prior and the noninformative prior are shown in Figure 7C for an exploration that takes 3 sensor readings per robot pose. The exploration is incomplete with unexplored areas shown in grey. Grey areas in the map that uses the proposed prior correspond to a probability of 0.9 and in the map produced using the non-informative prior to 0.5 , providing no information about the environment. This is an advantage of using the proposed prior since, even if areas remain unexplored, there is some information about what we expect to find there. The map that uses the proposed prior has correctly detected walls at the bottom of the image as well as the outer right wall. Conversely, using the non-informative prior has resulted in poorly detected outer walls and multiple grey areas that provide no information about the environment.

Figure 7D shows a comparison of the evolution of the $F_{2}$ metric with time when using the proposed informative prior and the non-informative prior when 3 sensor readings are used per pose and when only 1 reading is used per pose. These results agree with the simulation results, with the proposed method yielding a superior $F_{2}$ value even when a single sensor reading is used at each location. 

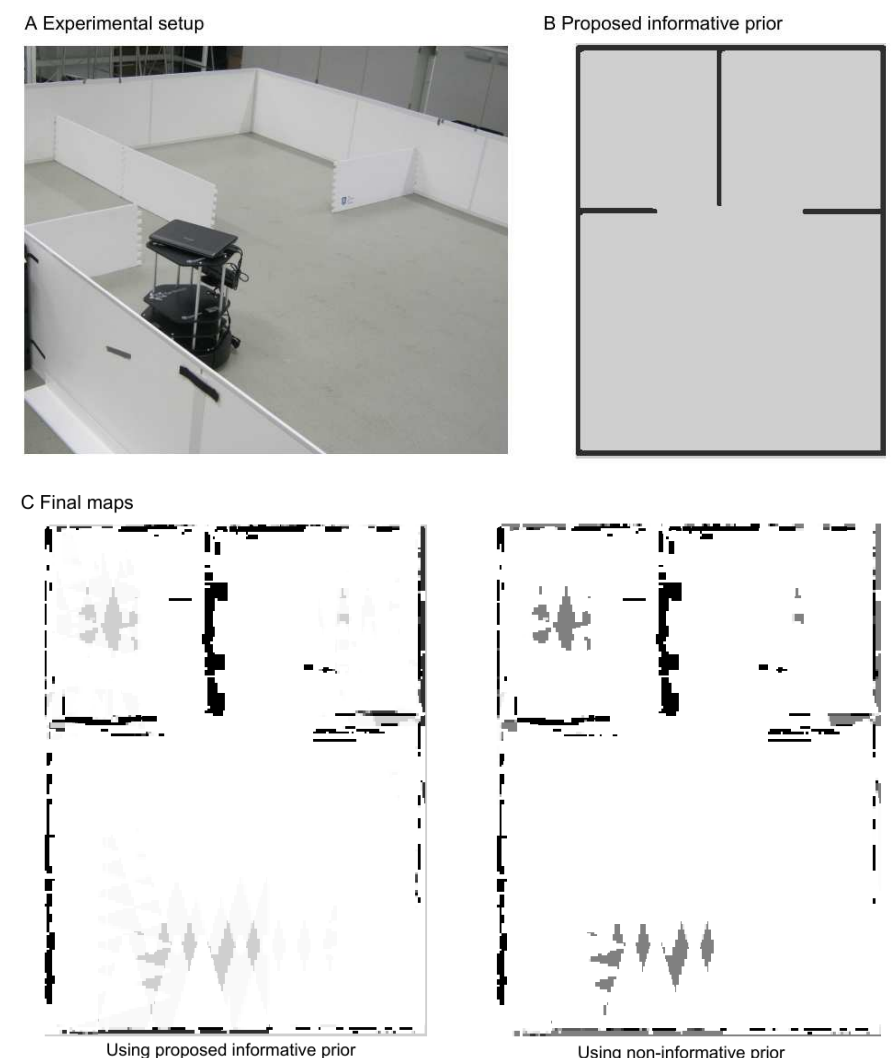

D Evolution of F2 metric with time
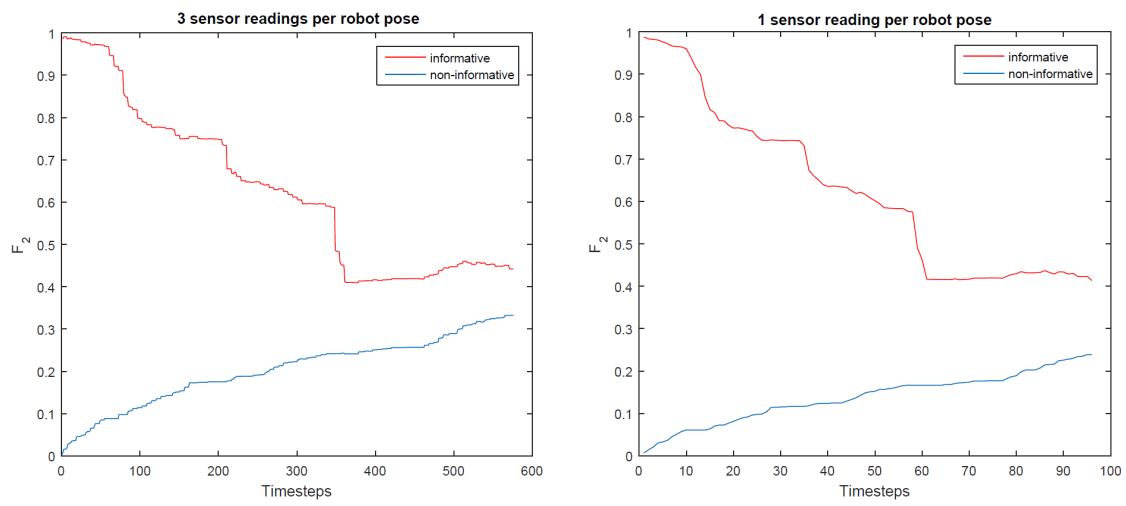

Figure 7: Experimental setup and results. A Experimental setup and robot used; B Accurate prior map of the environment; C Final maps produced using the proposed informative prior (left) and the non-informative prior (right); D Evolution of the $F_{2}$ metric with time for an exploration that takes 3 measurements at each robot pose (left) and for one that takes only 1 (right) using the informative and non-informative priors. 
Note that the starting $F_{2}$ value for the maps produced using the informative prior is very high because the prior used is very accurate and drops as less accurate sensor readings are incorporated. Even if the prior information is extracted accurately the final map should be updated using sensor information since floor plans may be outdated (for example due to a demolished wall) or no longer accurate (due to a collapsed wall in a USAR environment).

The multi-objective optimisation proposed in Section 3.3 was repeated for a more realistic sensor model and using the real Kinect data collected during the experiment. The following model was used, based on (Pirker et al. 2011)

$$
\begin{gathered}
\text { InverseSensorModel }\left(\mathbf{m}_{i}, \mathbf{x}_{k}, \mathbf{z}_{k}\right)= \\
\left\{\begin{array}{l}
0.9, \quad n_{i}^{\text {pixels }<z}=n_{i}^{\text {pixels }} \\
0.1, \quad n_{i}^{\text {pixels }>z}=n_{i}^{\text {pixels }} \\
\frac{n_{i}^{\text {pixels }<z}}{n_{i}^{\text {pixels }}}, \quad 0<n_{i}^{\text {pixels }<z}<n_{i}^{\text {pixels }}
\end{array}\right.
\end{gathered}
$$

with $n_{i}^{\text {pixels }<z}$ being the number of map pixels for a cell $i$ that fall between the robot and the location of the detected obstacle, $z, n_{i}^{\text {pixels }>z}$ the number of map pixels for a cell $i$ that are located beyond the detected object or at the location of the detected object and $n_{i}^{\text {pixels }}$ the number of map pixels in cell $i$. For an accurate sensor, cells for which all map pixels correspond to detected empty space are assigned a value of 0.9 , those that correspond to detected objects a value of 0.1 and those that contain both empty space and objects are assigned a value equal to the percentage of pixels corresponding to detected objects.

A very narrow range of optimal $\left(p_{\text {wall }}, p_{\text {space }}\right)$ was observed, which mostly agree with the results obtained in simulation, yielding opimised values of $p_{\text {wall }}=$ 0.2 and $p_{\text {space }}=1$. These values are multiplied by a factor of 0.9 before they are used in the prior map as explained in Section 5.1 to avoid values too close to 1 . A less accurate sensor model assigning 0.8 to detected empty space and 0.2 to detected walls was also tested, yielding optimised values $p_{\text {wall }}=0.4$ and $p_{\text {space }}=0.75$.

\subsection{Larger scale experimental results}

A larger scale experiment was conducted, mapping a floor of one of the University of Sheffield Engineering buildings (approximately 43x10 metres). The Matlab version of ROS Adaptive Monte Carlo Localisation (AMCL) was used alongside the floor plan of the building to produce estimates of the robot pose. The map was then updated using both the proposed informative prior and the commonly used non-informative prior. The floor plan was used for localisation in both cases to allow a direct comparison of the effects of different priors on mapping without taking into account the effects of the prior on localisation quality. Not using the floor plan to localise would lead to a less accurate map for the non-informative prior, which highlights a further advantage of using the 
prior map: more accurate localisation and the ability to start with a known, accurate $\mathbf{x}_{0}$.

The walls used to construct the prior map in this case, Figure $8 \mathrm{~A}$ are not perfectly extracted, with some of the doors appearing as wall sections on the 6 th room in the top row and the 5th room in the bottom row of offices. The final map is unaffected by these small inaccuracies which are corrected during mapping.

Some experiments were also run using a less accurate $\mathbf{x}_{0}$, using particles initialised with a non-zero variance. The resulting maps were less accurate but still better than those proposed using a non-informative prior. For a low variance the results were very similar to those produced using a perfectly accurate $\mathbf{x}_{0}$. Assuming a known prior pose is realistic since, given the extracted prior and a floor plan an operator can align the robot to a known location before starting exploration.

The results of these experiments are shown in Figure 8. Using the proposed prior yields a more accurate map both in a qualitative and quantitative sense. The map produced using the proposed prior yields a higher $F_{2}$ value, even if parts of the building are unexplored. It also yields more correctly mapped walls, demonstrating the advantages of using the proposed prior over a non-informative one. These results also demonstrate an added benefit of using a prior map. If exploration of the whole floor were not possible due to, for example, limited exploration time in a time-critical mission or certain areas being inaccessible, the proposed prior provides some information about unexplored areas. This may not be completely accurate but it would allow a human operator or rescuer to better interpret the mapped sections.

The map shown in Figure 8B is less easy to interpret: it is unclear what sections of the building the map areas correspond to and many of the walls have not been mapped correctly. Conversely, using the proposed informative prior, Figure $8 \mathrm{C}$, a human can interpret the map more easily. Moreover the majority of corridor walls have been mapped correctly, unlike Figure $8 \mathrm{~B}$ where many of them are mapped as empty space.

In all cases there appears to be some noise in the map, particularly within the detected rooms. That is due to the fact that the environment explored was a real world, cluttered office building, containing furniture. Therefore objects detected within the rooms mostly correspond to desks, chairs, bookcases and half-open doors. Since our aim is to improve performance in real world environments we did not aim to simplify the environment by only exploring long corridors (as is often done in the literature) or emptying the rooms.

These results further confirm that using the proposed informative prior can improve performance especially when a quick exploration is required, yielding consistently better quantitative and qualitative results. 


\section{A Extracted walls}

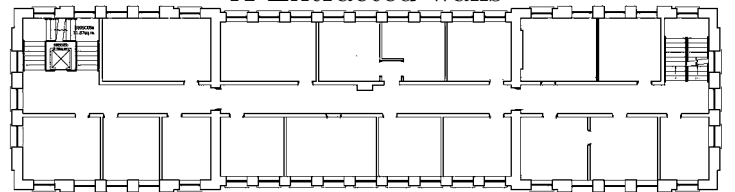

B Map produced using a non-informative prior

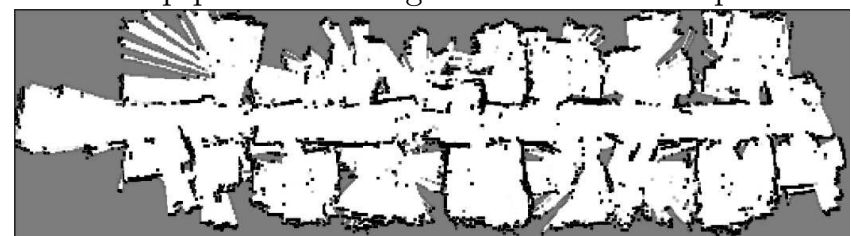

C Map produced using the proposed informative prior

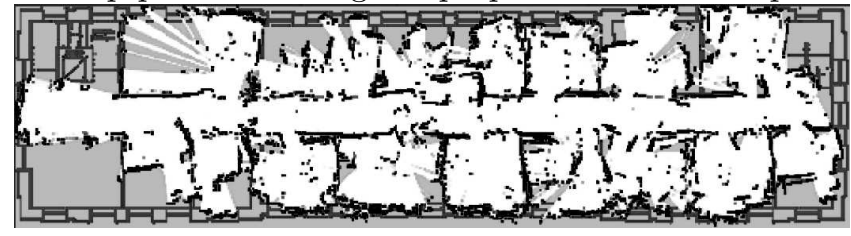

D Map produced using the proposed informative prior; $\mathbf{x}_{0}$ with a variance of 0.1 (less accurate initial pose)

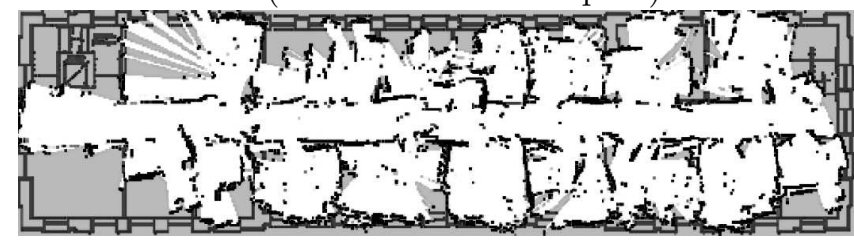

E Evolution of $F_{2}$ with time

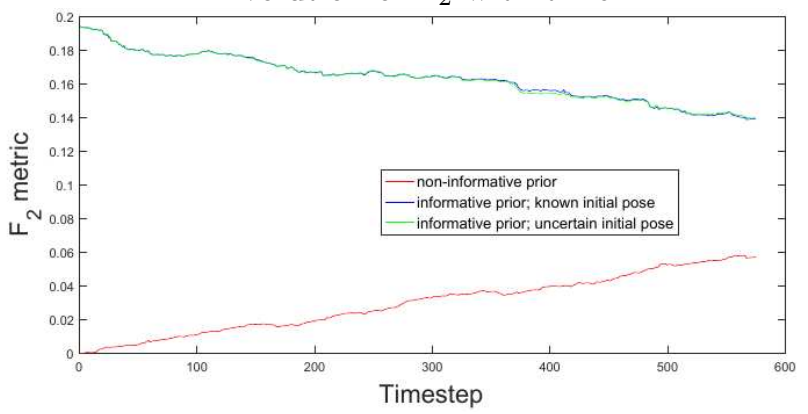

Figure 8: Large scale experimental results. A Prior map of extracted walls and empty space of Floor C of the Amy Johnson building, Dept of Automatic Control and Systems Engineering, University of Sheffield; B Map produced using a non-informative prior; C Map produced using the proposed prior, successfully mapping more walls than using the non-informative prior; D Map produced using the proposed informative prior and a slightly less accurate $\mathbf{x}_{0}$; E Evolution of the $F_{2}$ metric with time for an informative and non-informative prior. 


\section{Conclusions}

This paper presents a method to construct optimised indoors occupancy grid mapping priors. Priors were constructed using structural information extracted from architectural drawings and floor plans by assigning appropriate prior probabilities to detected wall and empty space locations. A precision-recall analysis was used to assess quantitative performance and a multi-objective optimisation was used to short list Pareto optimal solutions. Both types of drawings were found to have a similar region of $\left(p_{\text {wall }}, p_{\text {space }}\right)$ that yields good performance metrics.

The commonly used uninformative prior was found to perform worse than a prior constructed using an architectural drawing and was not one of the Pareto optimal solutions. The values $p_{\text {wall }}=0.2, p_{\text {space }}=0.9$ were found to yield optimal results in terms of qualitative performance whilst also yielding an improvement in $F_{2}$ metric of over $20 \%$.

The main contribution of this paper is thus a method to produce optimised Bayesian priors that improve map quality in indoors SLAM applications without adding to the computational complexity of the SLAM algorithm itself. There is a one-off cost of extracting the prior value but no further increase in computational complexity. This method also yields improved performance compared to the non-informative prior for coarser grids or explorations that do not cover the entire building. It is therefore very well suited to time critical challenging real life applications such as USAR missions where a fast and accurate SLAM implementation is required.

\section{Acknowledgments}

We would like to thank Jon Lipsky, lead developer of Elevenworks LLC, for allowing us to use a floor plan featured in the Elevenwork website, Figure 3 drawing (a) and the University of West Florida for allowing us to use an image of a floor plan of the Heritage Halls of residence, Figure 3 drawing (b) to test the mapping algorithms presented in this paper.

\section{Funding}

The research presented in this paper was funded by the University of Sheffield and PA Consulting.

\section{Appendix: Occupancy grid mapping}

The occupancy grid mapping algorithm (Elfes 1989) splits the environment to be mapped into a grid of cells and a prior probability is assigned to each cell. Cells are assumed to be independent in order to update the probability of occupancy of each grid cell. This assumption allows the factorisation 


$$
p\left(\mathbf{m} \mid \mathbf{X}_{0: k}, \mathbf{Z}_{0: k}\right)=\prod_{i} p\left(\mathbf{m}_{i} \mid \mathbf{X}_{0: k}, \mathbf{Z}_{0: k}\right)
$$

where $i=1, \ldots, N$ is the current grid cell and $N$ the total number of grid cells, making this an easier estimation since updating the probability for each cell given the robot pose and sensor readings is merely an update of the probability of occupancy of that cell. As the robot moves through the environment the probabilities of occupancy of cells that are within the field of view of the robot sensors are updated.

The probability that a cell $\mathbf{m}_{i}$ is occupied given the observation history is given by

$$
p\left(\mathbf{m}_{i} \mid \mathbf{Z}_{0: k}\right)=\frac{p\left(\mathbf{z}_{k} \mid \mathbf{m}_{i}, \mathbf{Z}_{0: k-1}\right) p\left(\mathbf{m}_{i} \mid \mathbf{Z}_{0: k-1}\right)}{p\left(\mathbf{z}_{k} \mid \mathbf{Z}_{0: k-1}\right)}
$$

which can be written as

$$
p\left(\mathbf{m}_{i} \mid \mathbf{Z}_{0: k}\right)=p\left(\mathbf{z}_{k} \mid \mathbf{m}_{i}\right) \frac{p\left(\mathbf{m}_{i} \mid \mathbf{Z}_{0: k-1}\right)}{p\left(\mathbf{z}_{k} \mid \mathbf{Z}_{0: k-1}\right)}
$$

using the static world assumption which states that past sensor readings are conditionally independent given knowledge of the map $\mathbf{m}$ (Thrun 2003). Thus Equation 18 can be written as

$$
p\left(\mathbf{m}_{i} \mid \mathbf{Z}_{0: k}\right)=\frac{p\left(\mathbf{m}_{i} \mid \mathbf{z}_{k}\right) p\left(\mathbf{z}_{k}\right)}{p\left(\mathbf{m}_{i}\right)} \frac{p\left(\mathbf{m}_{i} \mid \mathbf{Z}_{0: k-1}\right)}{p\left(\mathbf{z}_{k} \mid \mathbf{Z}_{0: k-1}\right)}
$$

using $p\left(\mathbf{z}_{k} \mid \mathbf{m}_{i}\right)=\frac{p\left(\mathbf{m}_{i} \mid \mathbf{z}_{k}\right) p\left(\mathbf{z}_{k}\right)}{p\left(\mathbf{m}_{i}\right)}$.

Placing Equation 19 in the odds form we get

$$
\begin{array}{r}
\operatorname{odds}\left(\mathbf{m}_{i} \mid \mathbf{Z}_{0: k}\right)=\frac{p\left(\mathbf{m}_{i} \mid \mathbf{Z}_{0: k}\right)}{p\left(\neg \mathbf{m}_{i} \mid \mathbf{Z}_{0: k}\right)} \\
=\frac{p\left(\mathbf{m}_{i} \mid \mathbf{z}_{k}\right) p\left(\mathbf{m}_{i} \mid \mathbf{Z}_{1: k-1}\right) p\left(\neg \mathbf{m}_{i}\right)}{p\left(\neg \mathbf{m}_{i} \mid \mathbf{z}_{k}\right) p\left(\neg \mathbf{m}_{i} \mid \mathbf{Z}_{1: k-1}\right) p\left(\mathbf{m}_{i}\right)}
\end{array}
$$

Therefore

$$
\begin{aligned}
& \operatorname{odds}\left(\mathbf{m}_{i} \mid \mathbf{Z}_{0: k}\right)= \\
& \operatorname{odds}\left(\mathbf{m}_{i} \mid \mathbf{z}_{k}\right) \operatorname{odds}\left(\mathbf{m}_{i} \mid \mathbf{Z}_{1: k-1}\right)\left(\operatorname{odds}\left(\mathbf{m}_{i}\right)\right)^{-1}
\end{aligned}
$$

where $p\left(\mathbf{m}_{i}\right)$ is the prior probability of occupancy of the $i$ th grid cell, odds $\left(\mathbf{m}_{i} \mid \mathbf{Z}_{1: k-1}\right)$ is the odds at the previous time step and $\operatorname{odds}\left(\mathbf{m}_{i} \mid \mathbf{z}_{k}\right)$ represents the inverse sensor model. Taking the logarithm of Equation 21, the log odds representation of Equation 21 is

$$
\mathbf{l}_{k, i}=\text { InverseSensor Model }\left(\mathbf{m}_{i}, \mathbf{x}_{k}, \mathbf{z}_{k}\right)+\mathbf{l}_{k-1, i}-\mathbf{l}_{0, i}
$$


with

$$
\mathbf{l}_{k, i}=\log \frac{p\left(\mathbf{m}_{i} \mid \mathbf{Z}_{1: k}, \mathbf{x}_{1: k}\right)}{1-p\left(\mathbf{m}_{i} \mid \mathbf{Z}_{1: k}, \mathbf{x}_{1: k}\right)}
$$

Methods to select a suitable or preferable inverse sensor model are proposed in (Thrun 2003) and (Yaqub \& Katupitiya 2007).

\section{References}

Arthur, D. \& Vassilvitskii, S. (2007), k-means++: The advantages of careful seeding, in 'Proceedings of the 18th Annual ACM-SIAM Symposium on Discrete algorithms', pp. 1027-1035.

Baden-Powell, C., Hetreed, J. \& Ross, A. (2011), Architect's Pocket Book, Elsevier.

Barnes, D., Maddern, W. \& Posner, I. (2015), Exploiting 3D Semantic Scene Priors for Online Traffic Light Interpretation, in 'Proceedings of the IEEE Intelligent Vehicles Symposium'.

Burschka, D., Geiman, J. \& Hager, G. (2003), Optimal landmark configuration for vision-based control of mobile robots, in 'Proceedings of the IEEE International Conference on Robotics and Automation', Vol. 3, pp. 3917-3922 vol.3.

Collins, T., Collins, J. \& Ryan, C. (2007), Occupancy Grid Mapping: An Empirical Evaluation, in 'Proceedings of the 15th Mediterranean Conference on Control and Automation'.

Davis, J. \& Goadrich, M. (2006), The relationship between Precision-Recall and ROC curves, in 'Proceedings of the 23rd International Conference on Machine Learning', ACM Press, pp. 233-240.

de la Puente, P. \& Rodriguez-Losada, D. (2014), 'Feature based graph-SLAM in structured environments', Autonomous Robots 37(3), 243-260.

Deb, K. (2001), Multi-objective optimization using evolutionary algorithms, Vol. 16, Wiley Interscience Series in Systems and Optimization.

Deb, K., Pratap, A., Agarwal, S. \& Meyarivan, T. (2002), 'A fast and elitist multiobjective genetic algorithm: NSGA-II', IEEE Transactions on Evolutionary Computation 6(2), 182-197.

Dissanayake, M., Newman, P., Clark, S., Durrant-Whyte, H. \& Csorba, M. (2001), 'A solution to the Simultaneous Localization And Map building (SLAM) problem', IEEE Transactions on Robotics and Automation 17(3), 229-241.

Durrant-Whyte, H. \& Bailey, T. (2006), 'Simultaneous Localization and Mapping : Part I', IEEE Robotics \& Automation Magazine 13(2), 99 - 110. 
Elfes, A. (1989), 'Using occupancy grids for mobile robot perception and navigation', Computer 22(6), 46-57.

Georgiou, C., Anderson, S. \& Dodd, T. (2015), Constructing contextual SLAM priors using architectural drawings, in 'Proceedings of the International Conference on Automation, Robotics and Applications', pp. 50-56.

Giagkiozis, I., Purshouse, R. C. \& Fleming, P. J. (2015), 'An overview of population-based algorithms for multi-objective optimisation', International Journal of Systems Science 46(9), 1572-1599.

Grewe, R., Komar, M., Hohm, A., Lueke, S. \& Winner, H. (2012), Evaluation method and results for the accuracy of an automotive occupancy grid, in 'Proceedings of the IEEE International Conference on Vehicular Electronics and Safety', pp. 19-24.

Hartigan, J. A. \& Wong, M. A. (1979), 'Algorithm AS 136: A K-Means Clustering Algorithm', Journal of the Royal Statistical Society. Series C (Applied Statistics) 28(1), pp. 100-108.

Kumar, V., Rus, D. \& Singh, S. (2004), 'Robot and Sensor Networks for First Responders', IEEE Pervasive Computing 3(4), 24-33.

Maddern, W., Pascoe, G. \& Newman, P. (2015), Leveraging Experience for Large-Scale LIDAR Localisation in Changing Cities, in 'Proceedings of the IEEE International Conference on Robotics and Automation'.

Mazuran, M., Tipaldi, G. D. \& Stachniss, C. (2014), A Statistical Measure for Map Consistency in SLAM, in 'Proceedings of the IEEE International Conference on Robotics and Automation', pp. 3650-3655.

Milstein, A. (2005), Occupancy Grid Maps for Localization and Mapping, in X.-J. Jing, ed., 'Motion Planning', InTech, chapter 19, pp. 381-408.

Montemerlo, M., Thrun, S., Koller, D. \& Wegbreit, B. (2003), FastSLAM 2.0: An Improved Particle Filtering Algorithm for Simultaneous Localization and Mapping that Provably Converges, in 'Proceedings of the International Joint Conferences on Artificial Intelligence'.

Parsley, M. \& Julier, S. (2011), Exploiting prior information in GraphSLAM, in 'Proceedings of the IEEE International Conference on Robotics and Automation', pp. 2638-2643.

Pirker, K., Rüther, M., Bischof, H. \& Schweighofer, G. (2011), Fast and accurate environment modeling using three-dimensional occupancy grids, pp. 11341140.

Powers, D. M. W. (2011), 'Evaluation: From Precision, Recall and F-Measure to ROC., Informedness, Markedness \& Correlation', Journal of Machine Learning Technologies 2(1), 37-63. 
Thrun, S. (2003), 'Learning Occupancy Grid Maps With Forward Sensor Models', Autonomous Robots 15(2), 111-127.

Williams, B. \& Reid, I. (2010), On combining visual SLAM and visual odometry, in 'Proceedings of the IEEE International Conference on Robotics and Automation', pp. 3494-3500.

Yaqub, T. \& Katupitiya, J. (2007), Laser scan matching for measurement update in a particle filter, in 'Proceedings of the IEEE/ASME International Conference on Advanced Intelligent Mechatronics', Ieee, pp. 1-6. 